\title{
Molecular dynamics simulations of wake structures behind a microparticle in a magnetized ion flow. I. Collisionless limit with cold ion beam
}

Cite as: Phys. Plasmas 25, 083702 (2018); https://doi.org/10.1063/1.5039587

Submitted: 09 May 2018 . Accepted: 17 July 2018 . Published Online: 01 August 2018

A. Piel, F. Greiner (D), H. Jung (D), and W. J. Miloch (D)
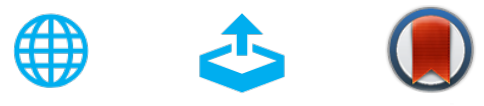

View Online

Export Citation

CrossMark

\section{ARTICLES YOU MAY BE INTERESTED IN}

Molecular dynamics simulations of wake structures behind a microparticle in a magnetized ion flow. II. Effects of velocity spread and ion collisions

Physics of Plasmas 25, 083703 (2018); https://doi.org/10.1063/1.5039606

Experiments on wake structures behind a microparticle in a magnetized plasma flow Physics of Plasmas 25, 073703 (2018); https://doi.org/10.1063/1.5040838

Charging of an irregularly shaped particle in the sheath of an rf plasma Physics of Plasmas 25, 073702 (2018); https://doi.org/10.1063/1.5038183

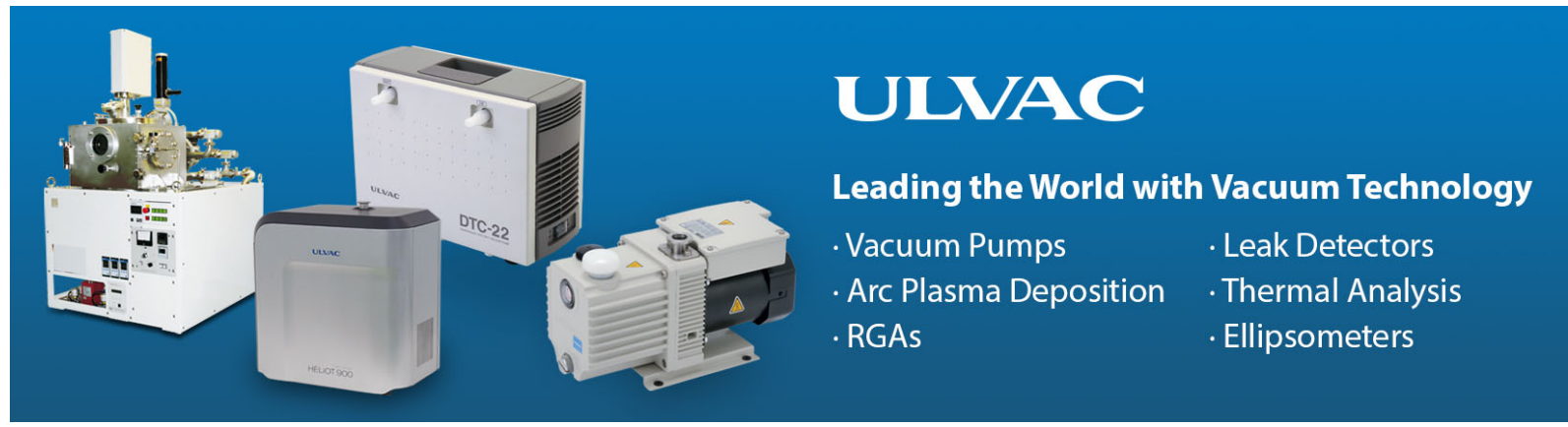




\title{
Molecular dynamics simulations of wake structures behind a microparticle in a magnetized ion flow. I. Collisionless limit with cold ion beam
}

\author{
A. Piel, ${ }^{1, a)}$ F. Greiner, ${ }^{1}$ H. Jung, ${ }^{1}$ and W. J. Miloch ${ }^{2}$ \\ ${ }^{1}$ Institute for Experimental and Applied Physics, Kiel University, D-24098 Kiel, Germany \\ ${ }^{2}$ Department of Physics, University of Oslo, Postboks 1048 Blindern, 0317 Oslo, Norway
}

(Received 9 May 2018; accepted 17 July 2018; published online 1 August 2018)

The potential and density structure behind a spherical microparticle in a magnetized ion flow are studied by means of molecular dynamics simulations. It is shown that, with increasing magnetization of the flow, the ion accumulation in the wake diminishes. Instead, ion depleted regions (shadows) form and ions accumulate at the edge of the shadows. The change of the ion density distribution also affects the force on other microparticles in the downstream region. For weak magnetization and a short distance, these interparticle forces can be attractive and non-reciprocal, as in the unmagnetized case. For large magnetization and further downstream, the force becomes repulsive. The mechanism of shadow formation is shown to involve a fast Coulomb scattering during a short fraction of the gyroperiod and subsequent trapping of the ions on large-radius gyro-orbits. Published by AIP Publishing. https://doi.org/10.1063/1.5039587

\section{INTRODUCTION}

The density and potential structure around spherical objects in magnetized plasmas are important problems in plasma physics, which are historically related to electric probes in laboratory plasmas ${ }^{1}$ and satellites in Earth orbit. ${ }^{2,3}$ When the plasma is flowing or when the satellite is moving at a velocity exceeding the ion-sound speed, the downstream side of the object shows complex wake potential structures as well as accumulation or depletion of charge. Intense studies of wake phenomena in unmagnetized situations were made in complex plasmas, where charged wakes can lead to attractive ${ }^{4-6}$ and non-reciprocal forces. ${ }^{7-10}$ A substantial accumulation of positive ion charge in unmagnetized particle wakes was found for ion speeds exceeding the Bohm speed $v_{\mathrm{B}}=\left(k_{\mathrm{B}} T_{\mathrm{e}} / m_{\mathrm{i}}\right)^{1 / 2}$, defined by electron temperature $T_{\mathrm{e}}$, Boltzmann's constant $k_{\mathrm{B}}$, and ion mass $m_{\mathrm{i}}$, while the effect becomes weaker for subBohm speeds and vanishes at $v \approx 0.3 v_{\mathrm{B}} \cdot{ }^{11,12}$ The potential pattern in the wake can be understood as the downstream convection of ion acoustic waves that are excited by the collision of the ion stream with the obstacle. ${ }^{4}$ This surplus charge is the origin of the attractive forces on another negatively charged particle downstream of the first particle. The situation becomes even more complex when the second particle is located in the wake of an upstream particle, which leads to the modification of its net charge. ${ }^{13-15}$

For unmagnetized plasmas, the wake structure is generally well understood from theories ${ }^{16,17}$ and simulations, ${ }^{11,14,18,19}$ in which the microparticle is embedded in a streaming (quasineutral) plasma.

Magnetized plasma wakes are still little explored. Analytical results from linear response (LR) theory were discussed in Refs. 20 and 21. Recently, the vanishing of wake patterns for supersonic flows and the appearance of interference patterns on the upstream side were reported in computations based on LR theory. ${ }^{22}$ Since LR theory prescribes the charge

\footnotetext{
a)piel@physik.uni-kiel.de
}

on the particle, a self-consistent solution, in which the charge on a second particle is affected by the ion flow, ${ }^{13,23}$ requires simulation techniques. Particle-in-cell (PIC) simulations of magnetized wakes ${ }^{24,25}$ give the desired self-consistency of charge and forces but are limited to artificial mass ratios, which make comparisons with experiments difficult. In experiments with magnetized plasmas, ${ }^{26}$ it was found that the interparticle forces in a field-aligned pair of particles show a strong dependence on the magnetic induction $B$. There, it was conjectured that a new phenomenon, the formation of ion shadows, may be responsible for the observed changes of the interaction force. Other magnetized ion wake effects were reported for the alignment of microparticles with the wires of a metallic grid placed upstream. ${ }^{27}$

In this paper, a comprehensive study of the magnetized ion wake is presented in terms of molecular dynamics (MD) simulations. The streaming ions are represented by a shifted Maxwellian with a mean velocity $v=v_{\mathrm{B}}$ and a width given by the ion thermal speed. The situation with a shifted Maxwellian primarily applies to objects moving through a thermal plasma. This approximation is retained here for comparison with wake theories and simulations ${ }^{12,16-18,23}$ that made this approximation. The experimental situation, ${ }^{23,28,29}$ in which the microparticle is levitated in the sheath of a capacitive radio-frequency plasma, is more complex. The streaming velocity mimics only one aspect of the conditions in the plasma sheath, where our experiments are performed. Our simulations use absolute physical units and the values for particle radius, plasma density, and ion mass from experiments. A refined discussion, which includes the proper drift distribution, ion-neutral collisions, and a static electric field, can be found in Paper II. ${ }^{30}$

The MD code used here is very fast and can be run on a PC with a powerful graphics processing unit (GPU). This speed enhancement, however, comes at the expense of greater noise and a much smaller simulation volume. The main objective of the MD simulations is to gain an overview on a host of different situations for collisionless ion flows. 
In Sec. II, the mechanism of ion shadow formation is described within a simple model for Coulomb scattering in the presence of a magnetic field. In Sec. III, the MADBORIS code, which is the MD code used for this study, is described in detail. Section IV summarizes the results for the charging and the ion drag force on a single particle as well as the potential and ion density distribution in a magnetized supersonic ion flow. In Sec. V, the charging and the interparticle forces of two-particle systems are studied for horizontal and vertical displacement of the downstream particle. The results are summarized and discussed in Sec. VI.

\section{TRAJECTORIES OF MAGNETIZED IONS IN THE COULOMB FIELD}

For understanding the formation of a dynamic shadow, we first approach the problem analytically. A set of ion trajectories is considered in the combination of a magnetic field $\vec{B}$ with the Coulomb field of a small charged sphere of radius $r_{\mathrm{p}}$ and surface potential $\Phi_{\mathrm{p}}$. The equation of motion (EOM) for an ion of mass $m_{\mathrm{i}}$ and charge $q_{\mathrm{i}}$ reads

$$
m_{\mathrm{i}} \ddot{\vec{r}}=q_{\mathrm{i}}(\vec{E}+\dot{\vec{r}} \times \vec{B}),
$$

with the electric field given by $\vec{E}=\left(\Phi_{p} r_{p} / r^{3}\right) \vec{r}$. Normalizing the time scale $\tau=\omega_{\mathrm{ci}} t$ by the ion cyclotron frequency $\omega_{\mathrm{ci}}$ $=|q| B / m_{\mathrm{i}}$ and the space scale $\vec{\rho}=\vec{r} / r_{1}=\left(x^{\prime}, y^{\prime}, z^{\prime}\right)$ by the scale factor $r_{1}=\left[\left|q \Phi_{\mathrm{p}}\right| r_{\mathrm{p}} /\left(m \omega_{\mathrm{ci}}^{2}\right)\right]^{1 / 3}$, we obtain the universal EOM

$$
\frac{d^{2} \vec{\rho}}{d \tau^{2}}=\operatorname{sgn}\left(q \Phi_{\mathrm{p}}\right) \frac{\vec{\rho}}{\rho^{3}}+\operatorname{sgn}(q) \frac{d \vec{\rho}}{d \tau} \times \vec{e}_{z} .
$$

For an attractive force on the ion, we have $\operatorname{sgn}\left(q \Phi_{\mathrm{p}}\right)=-1$. The problem has rotational symmetry about the $z$-axis. The universal EOM has only two parameters which completely determine the trajectory: the normalized impact parameter $b / r_{1}$ and the normalized initial velocity vector. The initial conditions can be chosen without loss of generality in the $x-z$ plane. Assuming that at $z^{\prime}=-100$, the asymptotic limit $z^{\prime} \rightarrow-\infty$ has been sufficiently approached, and introducing a Mach number $M=v / v_{\mathrm{B}}$ by normalizing velocities by the Bohm speed, the initial speed becomes

$$
\left.\frac{d z^{\prime}}{d \tau}\right|_{z^{\prime}=-100}=\frac{M v_{\mathrm{B}}}{r_{1} \omega_{\mathrm{ci}}} .
$$

Here, we have neglected any initial transverse velocity component, which is justified for a small ion thermal speed, $v_{\text {th,i }} / v_{\mathrm{B}} \ll 1$.

Equation (2) is solved numerically by a 4th-order Runge-Kutta scheme with an adaptive step size. We choose $M=1$ and the surface potential as $\Phi_{\mathrm{p}}=-3.9 k_{\mathrm{B}} T_{\mathrm{e}} / e$ (e being the elementary charge), which is the orbital-motion limited (OML) value for $M=1$ in $\operatorname{argon} .^{31,32}$ The resulting trajectories for a set of ten impact parameters are plotted in Fig. 1(a) in cylindrical coordinates $(r, z)$ and the normalization parameter $r_{1}$ cancels in $r / r_{\mathrm{p}}=r^{\prime} / r_{\mathrm{p}}^{\prime}$.

All displayed trajectories are subject to strong scattering by the dust particle. A large, ion-free space is created behind
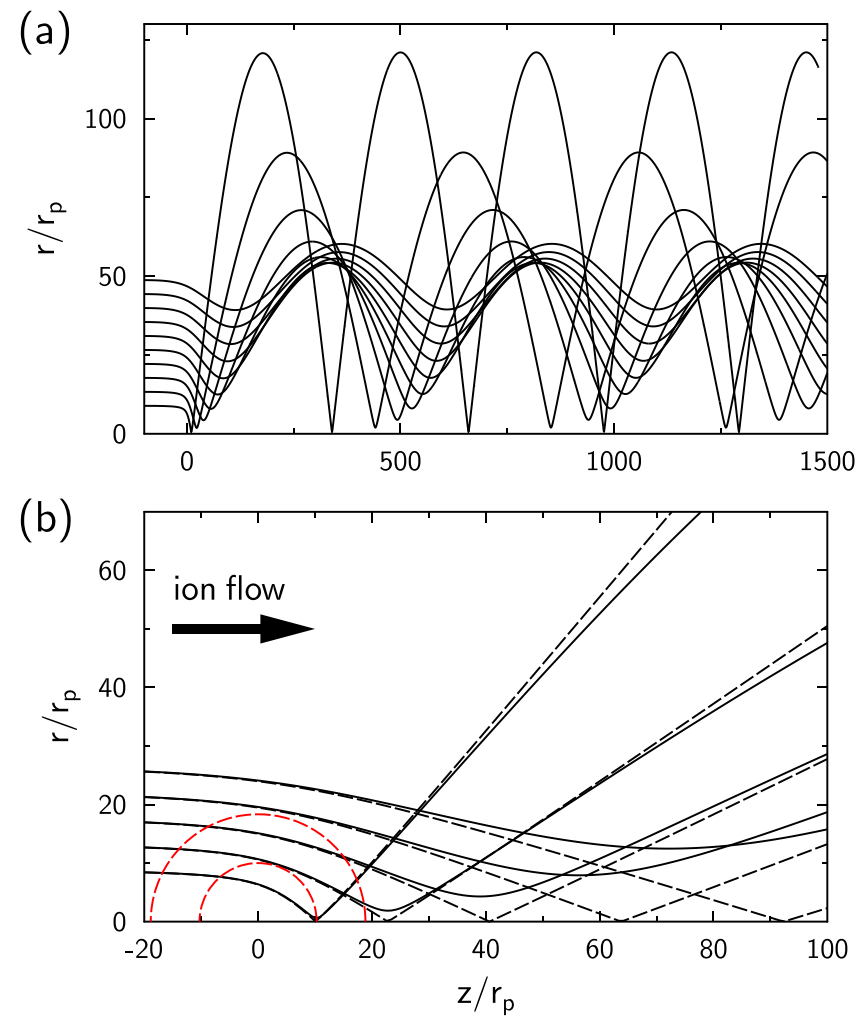

FIG. 1. (a) Ion trajectories in an attractive potential by a dust particle located at the origin $(r=z=0)$ for a Bohm Mach number $M=1$ and $B=1 \mathrm{~T}$. (b) Comparison of the scatter process with trajectories in the unmagnetized case (dashed lines). The dashed semi-circles about the origin illustrate the concept of a collision sphere for the two innermost trajectories.

the microparticle, which we name the dynamic ion shadow to distinguish from the static magnetic shadow behind large objects that is caused by absorption. This region has a fairly sharp edge represented by the envelope of the trajectories. The bunching of trajectories at the shadow edge leads to an enhanced ion density at the shadow edge. The dynamic shadow phenomenon forms a periodic sequence of ion-free spaces, which are separated by a periodic collapse of the shadow radius. The formation of a dynamic shadow had been already suggested in Ref. 26 and is also studied by us with particle-incell simulations. ${ }^{25}$

According to the considerations above, the formation of a dynamic shadow is an intrinsically nonlinear process that is governed by large-angle collisions of the ions with the microparticle. Therefore, dynamic shadows cannot be described by LR theory.

The formation of a dynamic shadow can be understood by the following argument of separated time scales. In the magnetized case, for $M=1$ and $T_{\mathrm{e}} \gg T_{\mathrm{i}}$, the ion motion is essentially along the magnetic field, which means that the Lorentz force practically vanishes. Therefore, the ion can approach the particle on its field line, which has an impact parameter $b$, until it enters a "collision sphere," see Fig. 1(b). The radius of this sphere is of the order of (1..2) $b$. Then, the Coulomb scattering takes place on a time-scale shorter than the gyroperiod. For the innermost two trajectories, this seems to be a good approximation, which is justified by the agreement with the unmagnetized trajectories (dashed 
curves). For increasing impact parameter, the deviations gradually become larger.

The scattering by a large angle in a short collision event leads to an effective transformation of energy from the axial motion to gyromotion. In this way, the strong scattering events in a magnetized ion stream can be decomposed into outer trajectory sections, which are governed by the conservation of energy and canonical momentum, ${ }^{1}$ and a nearly unmagnetized Rutherford scattering event within the collision sphere.

\section{THE MAD-BORIS SIMULATION CODE}

The single-ion model misses some important points. In the situation of a microparticle in a complex plasma, there are many ions simultaneously present in the vicinity of the microparticle, which lead to shielding of the particle charge and modified trajectories. Furthermore, the charge on the microparticle and the corresponding surface potential cannot be prescribed but need to be calculated from the ion and electron fluxes to the particle surface. Here, we present a MD approach for this situation.

The original "molecular asymmetric dynamics" (MAD) code $^{19}$ describes the motion of dressed ions in the field of an assembly of highly charged microparticles in the absence of a magnetic field. Comparisons with PIC simulations had shown that this asymmetric treatment gives a reasonable quantitative agreement for the potential distribution and interparticle forces.

The concept of asymmetric dynamics is illustrated by a cartoon (Fig. 2). It is based on the observation that the electron contribution to the shielding of dust grains cannot be described by the Debye-Hückel approximation, which assumes that the Boltzmann factor $\exp \left[-e \Phi(r) /\left(k_{\mathrm{B}} T_{\mathrm{e}}\right)\right]$ can be approximated by linearization. Here, $\Phi(r)$ is the electrostatic potential, $k_{\mathrm{B}}$ is the Boltzmann's constant, and $T_{\mathrm{e}}$ is the electron temperature. Rather, depending on the ion drift speed, a microparticle in a flowing plasma attains a negative floating potential $\Phi_{\mathrm{f}} \approx(-2.4 \cdots-3.9) k_{\mathrm{B}} T_{\mathrm{e}} / e .{ }^{31}$ Therefore,

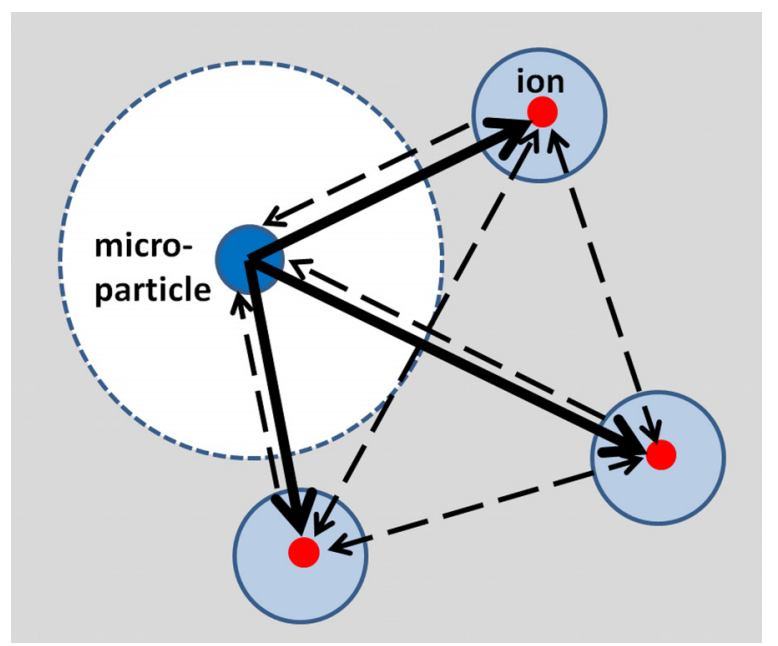

FIG. 2. The concept of asymmetric dynamics: The vicinity of the microparticle is nearly electron-free (white area). Ions are dressed with a sphere of enhanced electron density (blue shading). Solid arrows mark the naked Coulomb force, which the microparticle exerts on the ions; dashed arrows represent the shielded interaction force from ions. the electrons are efficiently removed from the immediate vicinity of the microparticle, where the large-angle collisions of ions occur that lead to charge accumulation in the wake. Consequently, the force which a microparticle exerts on other microparticles or on the ions is approximated by the "naked" Coulomb force (bold solid arrow). On the other hand, those ions which are not close to the microparticle fulfill the Debye-Hückel approximation and are considered as dressed ions, which interact with other ions and with microparticles (dashed arrows) by the Debye-Hückel (or Yukawa) potential

$$
\Phi_{\mathrm{Y}}(r)=\frac{e}{4 \pi \epsilon_{0} r} \exp \left(-\frac{r}{\lambda_{\mathrm{De}}}\right) .
$$

The shielding factor is given by the electron Debye length $\lambda_{\mathrm{De}}=\left(\epsilon_{0} k_{\mathrm{B}} T_{\mathrm{e}}\right)^{1 / 2}\left(n_{\mathrm{e} 0} e^{2}\right)^{-1 / 2}$ at the unperturbed electron density $n_{\mathrm{e} 0}$.

The force on a microparticle is the sum of all repulsive (from other microparticles) and attractive forces from the instantaneous "frozen" arrangement of the dressed ions. Therefore, we use a paradigm shift for the term "ion drag force." Originally, ${ }^{33}$ the orbital contribution to the ion drag was calculated from the momentum transfer in a completed ion collision. The concept of a sequence of ion collisions is now replaced by the net attraction of the microparticle by the geometrically deformed ion charge cloud.

The MAD-BORIS code, which is used in this paper, is a descendant for magnetized ion flows. Here, the Verlet particle pusher is replaced by a leap-frog Boris scheme. ${ }^{34}$ In the present investigations, ion-neutral collisions are neglected. The efficiency and accuracy of the Boris scheme were validated against the MAD code by setting $B=0$. The code uses a fast $N$-body routine derived from Ref. 35 that can handle typically $2^{17}$ superions and reaches $>60 \times 10^{9}$ pair interactions (single precision) per second with 2880 shaders. The simulation volume is a sphere of typically $R=3 \lambda_{\text {De }}$ radius. The ions in the simulation sphere are confined by a homogeneous background of shielded ions that fills the volume $r>R$ and leads to a confining potential $\Phi_{\mathrm{c}}$ of the form ${ }^{36}$

$$
\Phi_{\mathrm{c}}(r)=\frac{n_{\mathrm{i}} e \lambda_{\mathrm{De}}^{3}}{\epsilon_{0} r}\left(\frac{R}{\lambda_{\mathrm{De}}}+1\right) \mathrm{e}^{-R / \lambda_{\mathrm{De}}} \sinh \left(\frac{r}{\lambda_{\mathrm{De}}}\right) .
$$

Furthermore, the injection method for a spherical boundary ${ }^{37}$ is used. The number of simulation particles is $N_{\mathrm{s}}=2^{17}$, which results in a supercharge $Q=44 e$ of the simulation particles and a Coulomb coupling factor $\Gamma=Q^{2} /\left(4 \pi \epsilon_{0} a_{\mathrm{Ws}} k_{\mathrm{B}} T_{\mathrm{i}}\right)$ $=5.15$. Here, $a_{\mathrm{WS}}=\left(4 \pi n_{\mathrm{i}} / 3\right)^{-1 / 3}$ is the Wigner-Seitz radius.

The electron and ion temperatures are $k_{\mathrm{B}} T_{\mathrm{e}}=100 k_{\mathrm{B}} T_{\mathrm{i}}$ $=2.5 \mathrm{eV}$. Self-consistent charging of the microparticle is achieved by collecting positive ions when the trajectories intersect the microparticle radius and by continuously accumulating an electron current that is determined by the instantaneous surface potential of the microparticle. The electrons are assumed to have a Maxwellian velocity distribution. In the presence of a magnetic field, the electron flow to a spherical microparticle of radius $r_{\mathrm{p}}$ and surface potential $\Phi_{\mathrm{p}}$ is no 
longer isotropic. In the limit $B \rightarrow 0$, the surface of the microparticle $4 \pi r_{\mathrm{p}}^{2}$ determines the electron current

$$
I_{\mathrm{e}}=-4 \pi r_{\mathrm{p}}^{2} n_{\mathrm{e}} e\left(\frac{k_{\mathrm{B}} T_{\mathrm{e}}}{2 \pi m_{\mathrm{e}}}\right)^{1 / 2} \exp \left(\frac{e \Phi_{\mathrm{p}}}{k_{\mathrm{B}} T_{\mathrm{e}}}\right) .
$$

Here, $n_{\mathrm{e}}$ is the electron density and $m_{\mathrm{e}}$ is the electron mass. In the opposite limit, $B \rightarrow \infty$, electrons are strictly tied to the magnetic field lines and can only reach the cross-section of the microparticle from both sides. Then, the electron current is only half the value given by (6). The transition between these limiting cases is described by a simple model, ${ }^{38}$ which extends earlier considerations from probe theory. ${ }^{1}$

Ions of temperature $T_{\mathrm{i}}$ are injected with a drift velocity parallel to the magnetic field lines at $M=1$. The ion density $n_{\mathrm{i}}=10^{15} \mathrm{~m}^{-3}$ corresponds to conditions in the bulk plasma. The radius of the microparticle is chosen as $r_{\mathrm{p}}=12.5 \mu \mathrm{m}$ for comparison with experimental conditions. ${ }^{29}$ The particle radius is small compared to the electron Debye length $\lambda_{\text {De }}=0.372 \mathrm{~mm}$.

\section{THE SINGLE PARTICLE SYSTEM}

\section{A. Comparison with LR theory}

We start with comparing the structure of the wake potential in our MD simulations with the LR theory presented in Fig. 6 of Ref. 22 for $M=1$. In order to keep the nonlinear effects small, a particle radius $r_{\mathrm{p}}=2.5 \mu \mathrm{m}$ was chosen, which results in a self-consistent charge number $Z \approx-18000$. The density of argon ions was $n_{\mathrm{i}}=10^{15} \mathrm{~m}^{-3}$, $T_{\mathrm{e}} / T_{\mathrm{i}}=100$ and the magnetic field was varied to set the values of the magnetization parameter $\beta=\omega_{\mathrm{ci}} / \omega_{\mathrm{pi}}$, which is defined by the ratio of the ion cyclotron frequency and ion plasma frequency. A comparison of the potential structures along the $z$-axis is shown in Fig. 3.
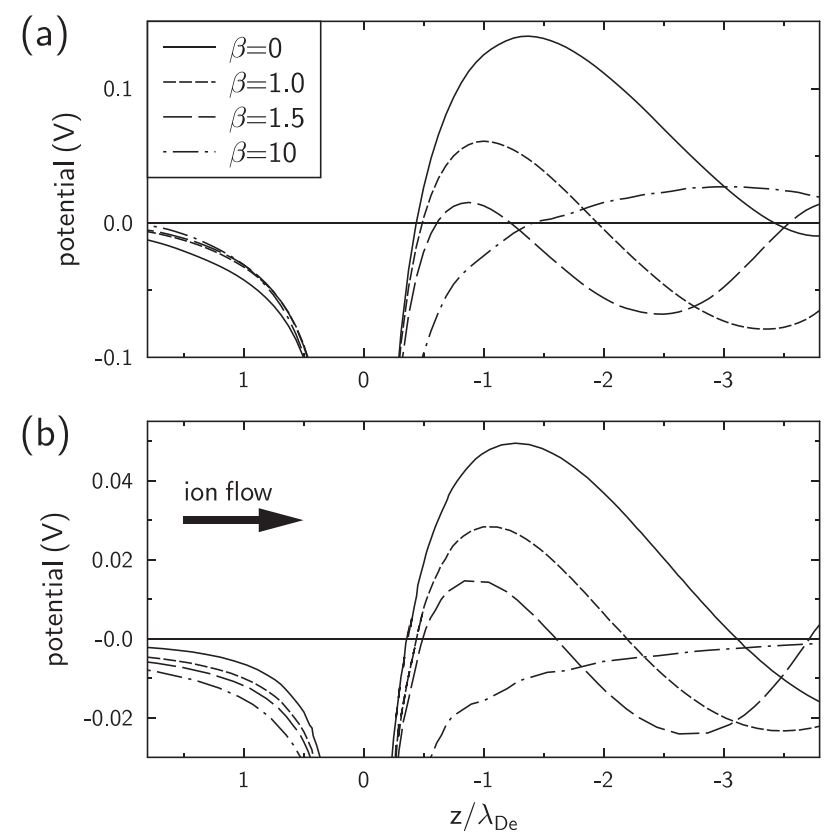

FIG. 3. Wake potential at $M=1$ for increasing magnetization parameter $\beta$ from (a) MD simulations and (b) LR theory (data from Fig. 6 of Ref. 22).
The MD simulations in panel (a) show an oscillatory potential in the wake $(z<0)$ of the particle located at $z=0$. For increasing magnetic field, the first positive maximum approaches the particle and the wavelength of the pattern gets shorter. At $\beta=10$, the oscillatory pattern vanishes. The LR theory in panel (b) shows the same behavior. The LR theory was calculated for $Z=-10000$ and the wave amplitude in the wake should increase $\propto Z$, i.e., by a factor of $\approx 1.8$. This general trend is observed, but the remaining small deviations may be related to nonlinear effects due to large angle collisions that determine ion focusing and shadow effects, which cannot be treated by LR theory. In summary, the general agreement of the wake structure with LR theory gives further confidence in the validity of the MD simulations.

\section{B. Density and potential structure}

The parameter studies are made for a large particle of $r_{\mathrm{p}}=2.5 \mu \mathrm{m}$ radius. The particle is placed at $x=0, z=1.0$ $\lambda_{\text {De }}$ to make best use of the simulation space for resolving the wake structure.

The potential and ion density distribution for this system are compiled in Fig. 4 for increasing magnetic induction. The unmagnetized limit is shown in panel (a). At $B=1 \mathrm{~T}$ in panel (b), positive ion charge is accumulated immediately downstream of the particle, but the total charge is smaller than in the unmagnetized limit. The wake potential attains a pronounced maximum at $z=-0.3 \lambda_{\text {De }}$. Outer potential contours approach the typical dovetail pattern of the unmagnetized case.

At $B=2 \mathrm{~T}$ in panel (c), the ion focus region is shifted towards the particle and the total surplus ion content of this region is further reduced. Different from panel (b), there is no longer a localized potential maximum in the wake. Rather, a wing-shaped positive contour remains. A new feature appears in the ion density with a cone-shaped region of strongly reduced ion density, which opens at $z=-0.4 \lambda_{\text {De }}$. We identify this region as the dynamic ion shadow that became already evident in Fig. 1. The ion density at the edge of the shadow is slightly enhanced above the average ion density and gives a v-shaped pattern that continues the "wings" of the positive charge region.

At $B=4 \mathrm{~T}$ in panel (d), the ion focus region is further compressed and the shadow region starts closing near the edge of the simulation region. The potential pattern has now changed and shows positive wings that match the enhanced ion density region near the edge of the shadow. At $B=10 \mathrm{~T}$ in panel (e), the ion focus region nearly vanishes. The shadow region now consists of two bubbles. Such a periodic structure in the shadow region was already expected from the single-ion scattering in Fig. 1(a) and is also observed in PIC simulations. ${ }^{25}$

A synopsis of the evolution of the ion density along the $z$-axis is shown in Fig. 5 for selected values of the magnetic induction. Note, how the density peak immediately downstream of the particle at $z=1.0 \lambda_{\text {De }}$ decreases and shifts towards the particle for increasing magnetic induction. The formation of the dynamic shadow becomes evident from density values below the average density. 

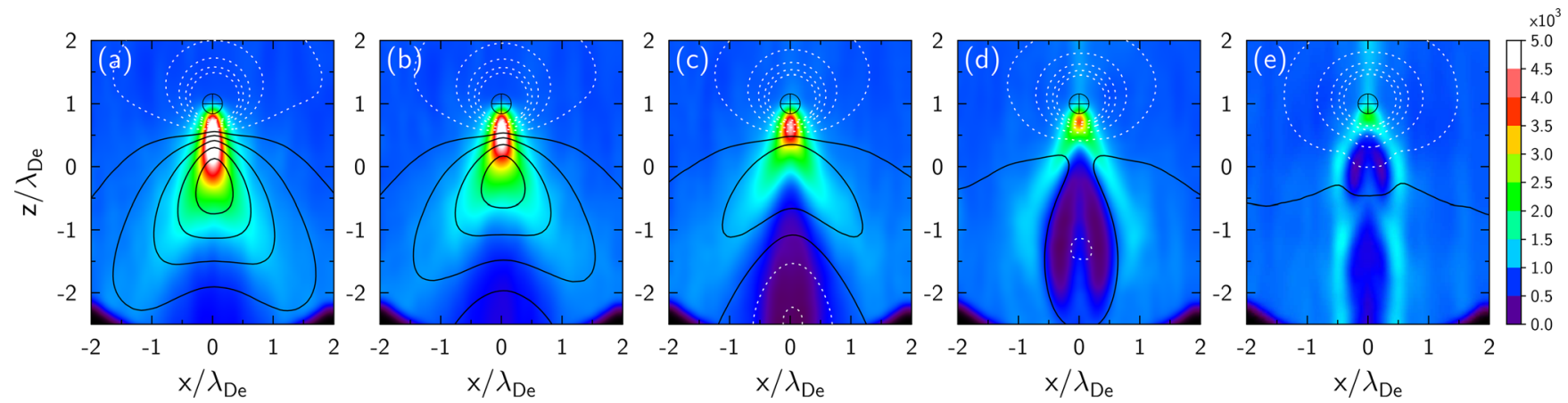

FIG. 4. Density of superions in units of $\lambda_{\mathrm{De}}^{-3}$ (color coded) and potential contours in $0.1 \mathrm{~V}$ steps: $\Phi<0$ (dotted), $\Phi \geq 0$ (full lines). (a) $B=0 \mathrm{~T}$, (b) $B=1 \mathrm{~T}$, (c) $B=2 \mathrm{~T}$, (d) $B=4 \mathrm{~T}$ (e) $B=10 \mathrm{~T}$. The particle position is marked with a $\oplus$ symbol. The exterior of the simulation volume $\left(r>3.0 \lambda_{\mathrm{De}}\right)$ appears as dark regions at the lower edges.

\section{Particle charge and drag force}

The evolution of the particle charge $q_{\mathrm{p}}$ and the ion drag force as a function of the magnetic induction are compiled in Fig. 6.

The particle charge at $B=0 \mathrm{~T}$ slightly exceeds the prediction from OML theory for a directed ion flow at $M=1$ in an unmagnetized plasma (dotted line). ${ }^{31}$ A detailed analysis of particle charging, based on PIC simulations in unmagnetized collisionless plasma flows, was given in Ref. 15. Since the OML model neglects shielding by ions, we note that shielding reduces the collection of positive ions, while the electron current remains unchanged, which may explain the observed overshoot. For $B=1 \mathrm{~T}$, the dust charge number attains its most negative value.

For increasing magnetic induction, the charge becomes gradually more positive and reaches saturation at $\approx 6 \mathrm{~T}$. The dominant physical mechanism behind this change is the reduction of the electron current by orbital motion. At $B=1$ $\mathrm{T}$ and $k_{\mathrm{B}} T_{\mathrm{e}}=2.5 \mathrm{eV}$, the thermal electron gyroradius reaches $r_{\mathrm{ce}}=5.3 \mu \mathrm{m}$ compared to the particle radius $r_{\mathrm{p}}=12.5 \mu \mathrm{m}$. For $B \gg 1 \mathrm{~T}$, the ratio $r_{\mathrm{ce}} / r_{\mathrm{p}}$ becomes small, which explains the substantial reduction of negative charge and the saturation. The magnitude of the ion drag force continuously diminishes for increasing magnetic induction.

In summary, essential changes of the wake structure occur in the range $B=(1-4) \mathrm{T}$, which is accessible in our experiments with argon plasmas. ${ }^{26,29}$

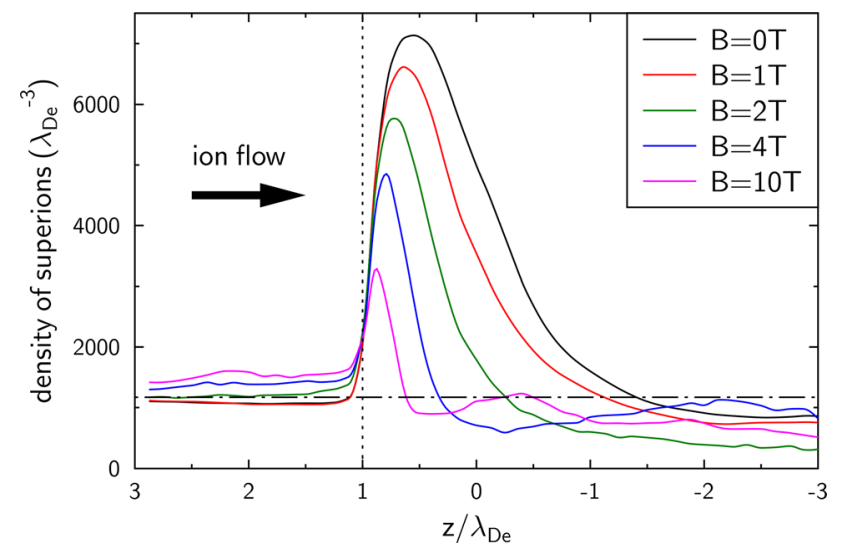

FIG. 5. Density of the superions along the $z$-axis for increasing magnetic induction. The particle is located at $z / \lambda_{\mathrm{De}}=1.0$. The dashed-dotted line marks the mean density of superions.

\section{TWO-PARTICLE SYSTEMS}

The density of superions along the $z$-axis is shown in Fig. 7 for a flow-aligned pair. In analogy to the experiments, we call the $z$ direction "vertical" and the $x$-direction "horizontal." The upstream (upper) particle is located at $z / \lambda_{\mathrm{De}}=1$ and the downstream (lower) particle at $z / \lambda_{\mathrm{De}}=0$. Ion charge accumulation on the downstream side of both particles is observed in all cases. For $B=1 \mathrm{~T}$, the density peak behind the lower particle is even higher than in the singleparticle situation shown in Fig. 5. This effect is due to the combined focusing action of the two particles and was already observed in the unmagnetized situation. ${ }^{19}$

For $B=2 \mathrm{~T}$, the ion density in the focus region of both particles becomes weaker (short-dashed curve). When the lower particle is shifted to $\Delta z=2.0 \lambda_{\mathrm{De}}$ the peak ion density behind the upstream particle remains unchanged (longdashed curve), whereas the peak behind the downstream particle is diminished.

\section{A. Horizontal shift of the downstream particle}

In the two-particle system, we are interested in potential and density distributions and their effect on the selfconsistent charging of both particles. The interparticle forces are studied for horizontal and vertical displacements of the particles.

\section{Potential and density distribution}

We begin with the situation at $B=1 \mathrm{~T}$ [Figs. 8(a)-8(d)], which according to the findings above can be considered as weakly magnetized. The upstream and downstream particles

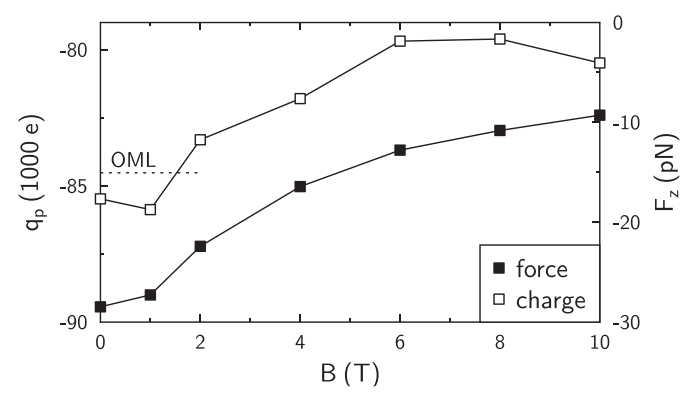

FIG. 6. Particle charge $q_{\mathrm{p}}$ and ion drag force $F_{z}$ for a single particle at $M=1$ as a function of the magnetic induction. 


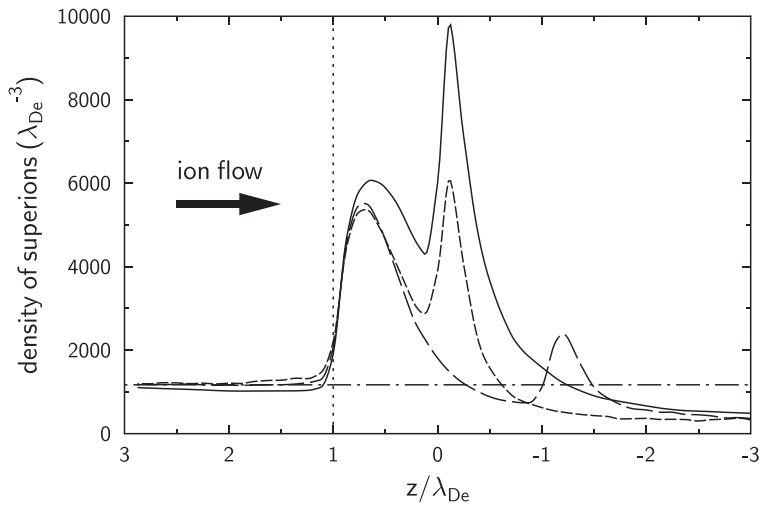

FIG. 7. Density of the superions along the $z$-axis in the vertically aligned two-particle system at $\Delta z=1.0 \lambda_{\mathrm{De}}$ for $B=1 \mathrm{~T}$ (full line), $B=2 \mathrm{~T}$ (shortdashed line), and $B=2 \mathrm{~T}$ at $\Delta z=2.0 \lambda_{\mathrm{De}}$ (long-dashed line). The upstream particle is located at $z / \lambda_{\mathrm{De}}=1$. The mean density of superions is marked by a horizontal dashed-dotted line.

have a vertical separation of $\Delta z=1.0 \lambda_{\mathrm{De}}$ and are symmetrically shifted in the horizontal direction. The particle positions are marked with $\oplus$ symbols.

The vertically aligned particle pair in panel (a) forms a connected region of enhanced ion density that fills the gap between the two particles. The positive potential contours demonstrate the existence of a positive potential hump below the downstream particle. The outer potential contours show the typical dovetail structure known from unmagnetized wakes. For $z<-2.0 \lambda_{\text {De }}$, the ion density is reduced below its unperturbed value, indicating the formation of a shadow region. For horizontally displaced particles, the positive ion clouds in panel (b) begin to split and become distinct in panels (c) and (d).

For $B=2 \mathrm{~T}$ and unchanged vertical separation, the ion density distribution and potential contours are shown in Figs. 8(e)-8(h). In the aligned position [panel (e)], the cloud sizes of enhanced ion density are smaller than at $B=1 \mathrm{~T}$ but still overlap. The two particles mostly act as a single combined particle that generates the v-shaped pattern of enhanced ion density with a shadow region for $z<1.5 \lambda_{\text {De }}$. Positive potential maxima are found outside of the v-shaped region. For larger separation in panel (f), the ion clouds still overlap and the entire vstructure is shifted to the right according to the position of the downstream particle. For even larger shifts in panels (g) and (h), the ion clouds become separated and individual v-shaped ion tails and separate shadow regions emerge.

The situation changes for a larger vertical separation of $\Delta z=2.0 \lambda_{\text {De }}$. The corresponding potential contours and ion density distribution are shown in Figs. 8(i)-8(m). Due to the larger vertical separation, the wake features of both particles are more clearly separated from the beginning. For $\Delta x=0$, the downstream particle resides inside the dynamic shadow of the other particle and forms its own dynamic shadow further downstream. In panels (k) and (l), the downstream particle crosses the $\mathrm{v}$-shaped region of enhanced ion density.

\section{Particle charge}

The evolution of the particle charge $q_{\mathrm{p}}$ as a function of the horizontal displacement is compiled in Fig. 9(a) for
$\Delta z=1.0 \lambda_{\mathrm{De}}, B=1 \mathrm{~T}$ and $B=2 \mathrm{~T}$. In both situations, the charge of the upstream particle (open symbols) is found nearly independent of the other particle's position. The downstream particle, however, shows a significant reduction of the negative charge for $\Delta x<\lambda_{\text {De }}$, when it is embedded in the cloud of enhanced ion density. This decharging effect becomes weaker at $B=2 \mathrm{~T}$ in accordance with the reduced ion accumulation.

The situation for $\Delta z=2.0 \lambda_{\mathrm{De}}$ and $B=2 \mathrm{~T}$ is shown in panel (b). Again, the upstream particle's charge is nearly constant. The downstream particle now shows a different evolution from panel (a). It starts in the dynamic shadow region, where it becomes considerably more negatively charged than the upstream particle. Decharging is found for $\Delta x=(0.5 \ldots 1.2) \lambda_{\mathrm{De}}$, when the downstream particle crosses the $\mathrm{v}$-shaped region of enhanced ion density in Figs. 8(1) and 8(m).

\section{Vertical interparticle forces}

The vertical interaction forces are shown in Figs. 10(a)-10(c) for the same situations as in Fig. 9. In panel (a), the net force on the upstream particle (open triangles) and downstream particle (full triangles) is shown at $\Delta z=1.0 \lambda_{\mathrm{De}}$, $B=1 \mathrm{~T}$. The dashed curves represent the sum of the ion drag on a single particle (marked by the dashed-dotted line) and the mutual Coulomb repulsion of the particle pair. This simple model captures the essential features of the interaction forces. The deviations from this simple model can be understood as nonlinear effects. The net force on the downstream particle is enhanced by the combined focusing action of the particle pair, whereas the force on the upstream particle is diminished.

For the same separation but $B=2 \mathrm{~T}$ in panel (b) the evolution of the forces is similar but the ion drag and the mutual repulsion are weaker due to the reduced negative charge. For a larger separation $\Delta z=2.0 \lambda_{\mathrm{De}}$ and $B=2 \mathrm{~T}$ in panel (c), the force evolution on the upstream particle remains flat due to the reduced repulsion and approaches the ion drag of the single particle. Surprisingly, the force on the downstream particle becomes even weaker than that on the upstream particle. This effect can be understood as an uplifting by the "missing ion charge" in the dynamic shadow region below.

\section{Horizontal interparticle forces}

Because, in the experiments, the two particles in a pair are confined to individual levitation heights, in which each of the particles is free to move, we are primarily interested in horizontal restoring forces which the downstream particle experiences from wake charges. The horizontal interaction forces are shown in Fig. 11.

For $\Delta z=1.0 \lambda_{\mathrm{De}}, B=1 \mathrm{~T}$ in panel (a), the horizontal force on the downstream particle (full triangles) is negative. This represents a restoring (attractive) force for a particle that is displaced in $+x$ direction. The force increases for $\Delta x \leq 0.4 \lambda_{\text {De }}$. Compared with the ion density distribution in Figs. 8(a) and 8(b), we expect this increasing force because the downstream particle is found inside the cloud of enhanced ion space charge. For $\Delta x>0.4 \lambda_{\text {De }}$, the horizontal force decreases again, as expected, because the particle is now outside of the cloud of enhanced ion density. This 



FIG. 8. Density of superions (color coded) and potential contours in $0.1 \mathrm{~V}$ steps (dotted: $\Phi<0$, full line: $\Phi \geq 0$ ) for a vertical separation of the two particles of $\Delta z=1.0 \lambda_{\mathrm{De}}$. (a)-(d) $B=1 \mathrm{~T}$, (e)-(h) $B=2 \mathrm{~T}$. (i) $-(\mathrm{m}) B=2 \mathrm{~T}$ but $\Delta z=2.0 \lambda_{\mathrm{De}}$. The ion density distribution is given in units of $\lambda_{\mathrm{De}}^{-3}$ and potential contours in $0.1 \mathrm{~V}$ steps. Dotted contours represent negative potentials; full lines mark zero and positive values. The exterior of the simulation volume $\left(r>3.0 \lambda_{\mathrm{De}}\right)$ appears as dark regions at the lower edges.

behavior is identical to the unmagnetized case. ${ }^{19}$ At $B=2 \mathrm{~T}$ (full circles), the horizontal force becomes weaker, but is still attractive.

The situation at $B=2 \mathrm{~T}$ changes completely when the vertical separation of the particles is enlarged to $\Delta z=2.0 \lambda_{\mathrm{De}}$ (full diamonds). Then the downstream particle is immersed in or becomes a horizontal neighbor of the upstream particle's shadow region and the horizontal force is repulsive.

Figure 11(b) shows that the horizontal force on the upstream particle is always negative for all three situations. Because of its negative excursion, this represents a repulsive force. As in the unmagnetized situation ${ }^{19}$ the horizontal forces turn out to be nonreciprocal, a typical feature for an open system with streaming ions.

\section{B. Vertical shift of the downstream particle}

In the following, we investigate the dependence of the vertical interaction force and particle charge on the interparticle distance and magnetic field intensity for particles aligned with the flow. The upstream particle is located at $z=2.0 \lambda_{\mathrm{De}}$. Typical ion density distributions and potential contours for $B=1 \mathrm{~T}$ and $B=2 \mathrm{~T}$ were already given in Figs. 8(a), 8(e), and 8(i).

The particle charges are compiled in Figs. 12(b) and 12(d). For both magnetic inductions, the charge of the upstream particle remains unaffected by the separation of the downstream particle and is very close to the charge of a single particle (dotted line). The charge on the downstream particle can be understood by comparing with the ion density distribution in Fig. 8 . At a close distance $\left(\Delta z \leq 2.0 \lambda_{\mathrm{De}}\right)$, a decharging of the downstream particle by the enhanced positive ion density from the focusing action of the upstream particle observed. The charge gradually becomes more negative and its magnitude exceeds the single-particle charge as soon as the downstream particle enters the dynamic shadow region, $\Delta z \geq 3.0 \lambda_{\text {De }}$. These two mechanisms explain the evolution of the charge of the downstream particle for $B=1 \mathrm{~T}$ and $B=2 \mathrm{~T}$. 

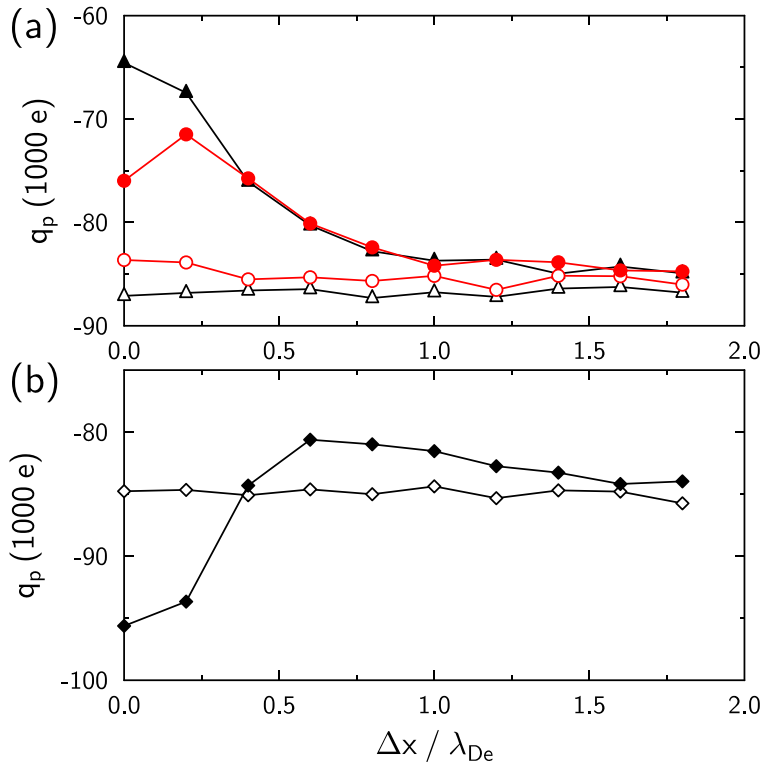

FIG. 9. Particle charge $q_{\mathrm{p}}$ as a function of horizontal displacement between upstream particle (open symbols) and downstream particle (full symbols). (a) $\Delta z=1.0 \lambda_{\mathrm{De}}, B=1 \mathrm{~T}$ (triangles), and $B=2 \mathrm{~T}$ (circles). (b) $\Delta z=2.0 \lambda_{\mathrm{De}}$, $B=2 \mathrm{~T}$.
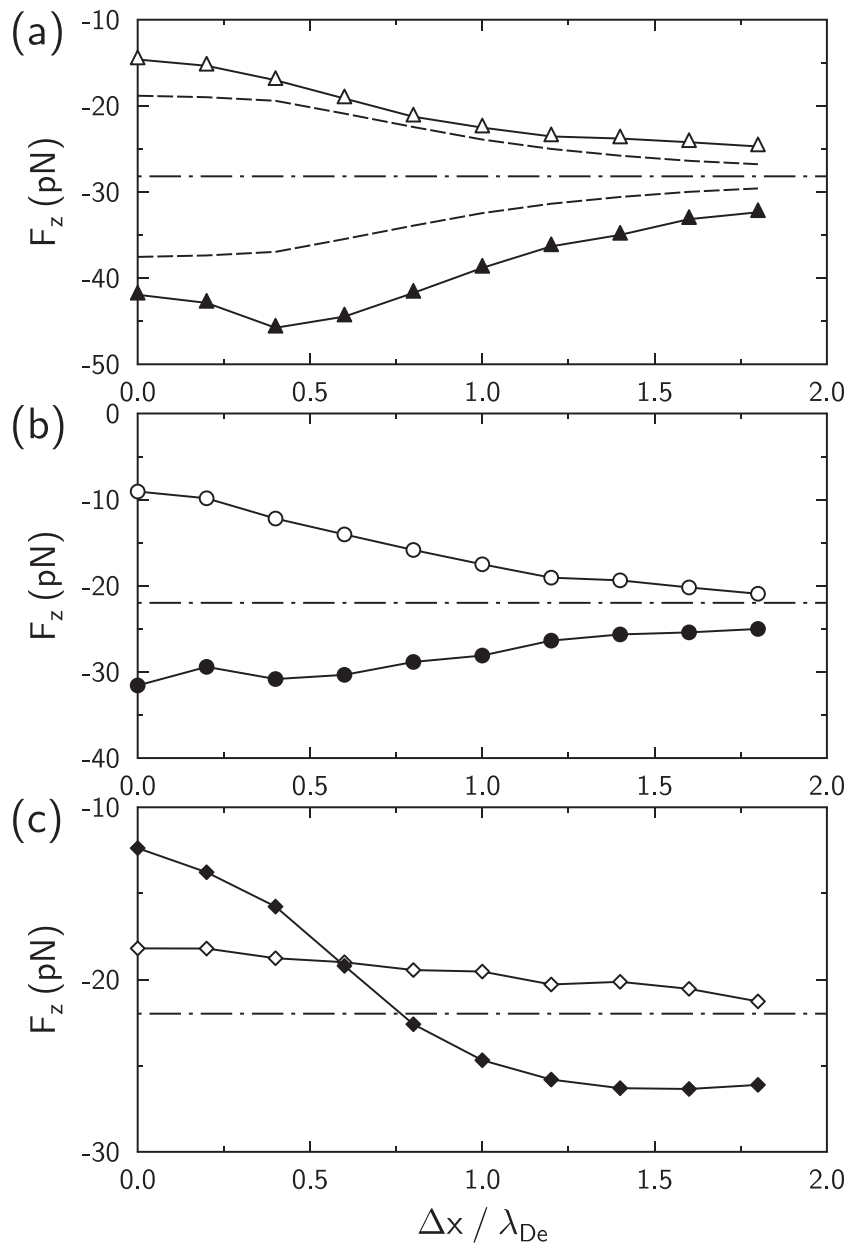

FIG. 10. Vertical interaction force as a function of horizontal displacement between upstream particle (open symbols) and downstream particle (full symbols). (a) $\Delta z=1.0 \lambda_{\mathrm{De}}, B=1 \mathrm{~T}$ (triangles) (b) $\Delta z=1.0 \lambda_{\mathrm{De}}$, and $B=2 \mathrm{~T}$ (circles). (c) $\Delta z=2.0 \lambda_{\mathrm{De}}, B=2 \mathrm{~T}$ (diamonds).
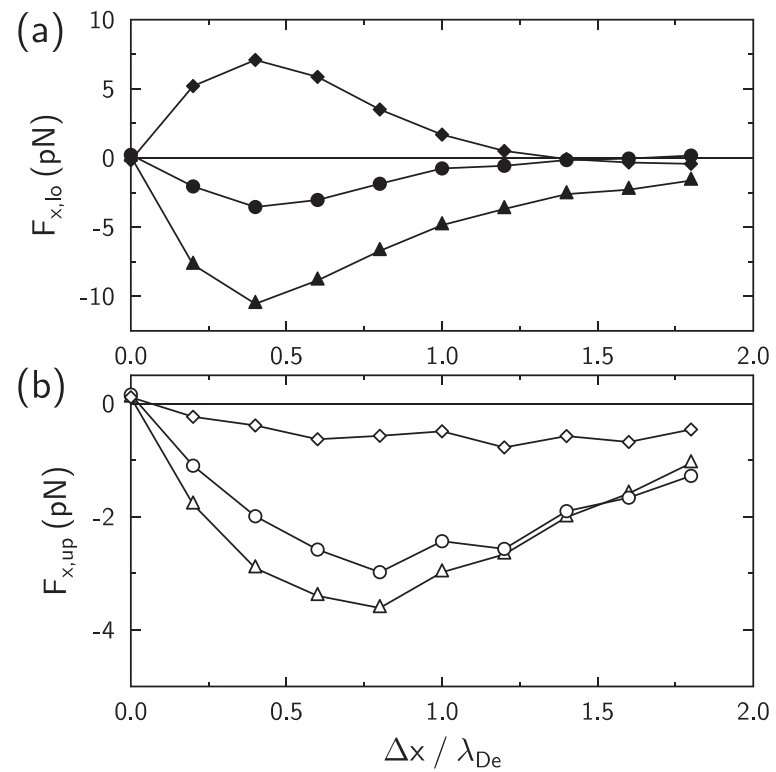

FIG. 11. (a) Horizontal force on the downstream particle (symbols as in Fig. 10). (b) Horizontal force on the upstream particle.

Because of the rotational symmetry about the magnetic field direction, the horizontal forces are practically zero. The vertical interaction forces during the vertical shift are compiled in Figs. 12(a) and 12(c). The upstream particle experiences a superposition of Coulomb repulsion by the lower particle and ion drag. For increasing separation $\Delta z$, the repulsive force on the upstream particle diminishes and approaches the typical ion drag force of an isolated particle (dash-dot line).

The magnitude of the force on the downstream particle diminishes for rising distance and even crosses the ion drag of the single particle and, at last, the force on the upstream particle. This crossing was attributed to the uplifting force from the deficit of ion charge in the dynamic shadow region. For $B=2 \mathrm{~T}$, this crossing occurs at smaller separation of the particles. This supports the given explanation because the dynamic shadow is now closer to the particle.
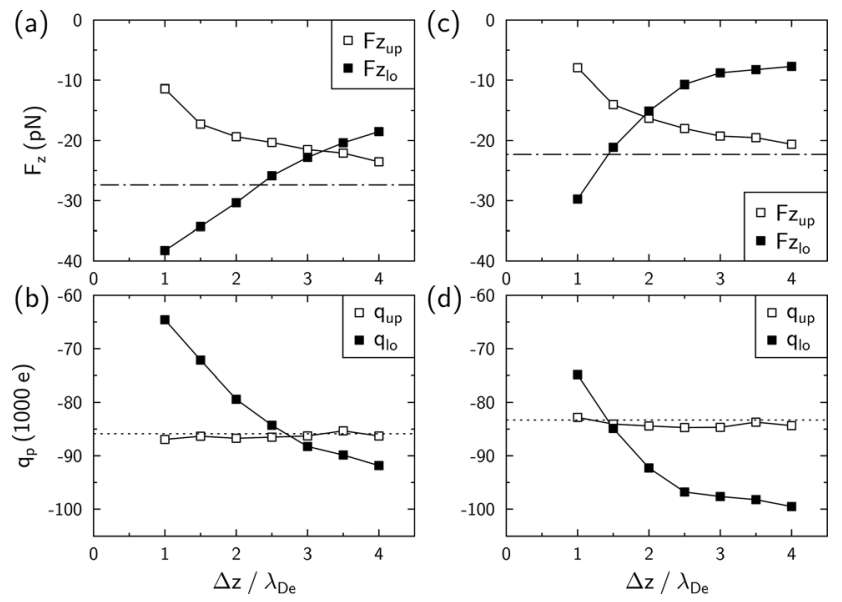

FIG. 12. Interaction force and particle charge during a vertical shift of the downstream particle. (a) and (b) $B=1 \mathrm{~T}$. (c) and (d) $B=2 \mathrm{~T}$. For reference, the force on a single particle is marked by a dotted-dashed line, the charge of a single particle by a dotted line. 


\section{DISCUSSION AND CONCLUSIONS}

The origin of the dynamic shadow phenomenon could be demonstrated by a study of the normalized trajectories in the combination of a radial Coulomb field with a superimposed axial magnetic field. The shadow is produced by efficient scattering of ions by the dust particles. These largeangle scattering events occur on a time-scale that is short compared to the gyroperiod and make the phenomenon a genuine non-linear process beyond the scope of LR theory.

MD simulations for a single microparticle show that the magnitude of the negative particle charge decreases for rising magnetic induction. This effect can mostly be attributed to the topological change from isotropic to field-aligned electron motion. For the same reason, the magnitude of the ion-drag force decreases. Furthermore, the ion focus region persists for moderate magnetization. For increasing magnetic induction, the focus region shrinks and moves closer to the particle, as was also observed in PIC simulations. ${ }^{24} \mathrm{~A}$ comparison with LR theory ${ }^{22}$ shows that the oscillatory potential structure in the wake shows the characteristic dependence on the magnetization parameter $\beta$.

As a new feature, a dynamic ion shadow appears further downstream. The shadow develops a periodic pattern in accordance with the single-ion model described in Sec. II and PIC simulations. ${ }^{25}$ The radial extension of the shadow and the scale length of its periodicity scale with $B^{-1}$.

Simulations with two particles show that for $B=1 \mathrm{~T}$ argon ions are still in a weakly magnetized situation. At $\Delta z=1.0 \lambda_{\text {De }}$, the horizontal force on a horizontally displaced downstream particle is found attractive as in the unmagnetized limit. This horizontal attractive force explains the experimental observation of alignment in particle pairs in the presence of strong magnetic fields. ${ }^{26}$ For higher magnetic field, $B=2 \mathrm{~T}$, this horizontal force diminishes by a factor of three but still remains attractive. However, at the larger vertical separation, $\Delta z=2.0 \lambda_{\text {De }}$, the downstream particle interacts horizontally with the ion shadow of the upstream particle and the horizontal force on the downstream particle becomes repulsive. In all situations, the horizontal force on the upstream particle is found repulsive. This makes the horizontal forces non-reciprocal, as in the unmagnetized case.

The vertical forces are a combination of mutual Coulomb repulsion and the attractive force from the complex anisotropic ion distribution. At large separation, the vertical force on the upstream particle converges to the single-particle ion drag force. For large separation, the negative force on the downstream particle can even be weaker than the net force on the upstream particle. This novel observation can be attributed to the uplifting force from the dynamic shadow region. The ion depletion of this region acts like a net negative charge, which pushes the downstream particle upwards.

The negative charge on the upstream particle remains unaffected by the other particle and remains close to the single-particle value. At small separation in a vertically aligned pair, the downstream particle is markedly decharged by the enhanced ion density in the focus region. When the downstream particle enters the shadow region, the negative charge becomes even larger than the single-particle value.
In summary, we have demonstrated how the external magnetic field can modify the potential and density distributions in the wake, as well as particle charging and interparticle forces. Ion shadows and their envelopes will lead to distinct decharging of downstream particles and equilibrium positions, which depends on the interparticle distances. A particle placed in the ion shadow region can acquire even larger charge than the upstream particle, which is distinct from unmagnetized plasmas, and will result in complex interactions between the particles. Thus, strong magnetization of plasma will affect interactions between dust particles, and in particular ordering of dust clusters in plasma flows.

\section{ACKNOWLEDGMENTS}

Part of the investigations at Kiel University was financially supported by DFG within the Transregional Collaborative Research Centre TR-24 (project A2). W.J.M. acknowledges funding from the Research Council of Norway (Project No. 240000).

${ }^{1}$ J. Rubinstein and J. G. Laframboise, Phys. Fluids 25, 1174 (1982).

${ }^{2}$ J. G. Laframboise and L. J. Sonmor, J. Geophys. Res. 98, 337, https:// doi.org/10.1029/92JA00839 (1993).

${ }^{3}$ D. E. Hastings, J. Geophys. Res. 100, 14457, https://doi.org/10.1029/ 94JA03358 (1995)

${ }^{4}$ M. Nambu, S. V. Vladimirov, and P. K. Shukla, Phys. Lett. A 203, 40 (1995).

${ }^{5}$ S. V. Vladimirov and M. Nambu, Phys. Rev. E 52, R2172(R) (1995).

${ }^{6}$ F. Melands $\varnothing$ and J. Goree, Phys. Rev. E 52, 5312 (1995).

${ }^{7}$ A. Melzer, V. Schweigert, I. Schweigert, A. Homann, S. Peters, and A. Piel, Phys. Rev. E 54, R46 (1996).

${ }^{8}$ V. A. Schweigert, I. V. Schweigert, A. Melzer, A. Homann, and A. Piel, Phys. Rev. E 54, 4155 (1996).

${ }^{9}$ K. Takahashi, T. Oishi, K. Shimomai, Y. Hayashi, and S. Nishino, Phys. Rev. E 58, 7805 (1998).

${ }^{10}$ A. Melzer, V. A. Schweigert, and A. Piel, Phys. Scr. 61, 494 (2000).

${ }^{11}$ W. Miloch, J. Trulsen, and H. Pécseli, Phys. Rev. E 77, 056408 (2008).

${ }^{12}$ I. H. Hutchinson, Phys. Rev. E 85, 066409 (2012).

${ }^{13}$ W. J. Miloch and D. Block, Phys. Plasmas 19, 123703 (2012).

${ }^{14}$ D. Block, J. Carstensen, P. Ludwig, W. J. Miloch, F. Greiner, A. Piel, M. Bonitz, and A. Melzer, Contrib. Plasma Phys. 52, 804 (2012).

${ }^{15}$ D. Block and W. J. Miloch, Plasma Phys. Controlled Fusion 57, 014019 (2015).

${ }^{16}$ P. Ludwig, W. J. Miloch, H. Kählert, and M. Bonitz, New J. Phys. 14, 053016 (2012).

${ }^{17}$ R. Kompaneets, A. V. Ivlev, V. Nosenko, and G. E. Morfill, Phys. Rev. E 89, 043108 (2014).

${ }^{18}$ I. H. Hutchinson, Phys. Rev. Lett. 107, 095001 (2011).

${ }^{19}$ A. Piel, Phys. Plasmas 24, 033712 (2017).

${ }^{20}$ M. Nambu, M. Salimullah, and R. Bingham, Phys. Rev. E 63, 056403 (2001).

${ }^{21}$ M. Salimullah, M. Tomey, P. K. Shukla, and A. K. Banerjee, Phys. Scr. 67, 534 (2003).

${ }^{22}$ J.-P. Joost, P. Ludwig, H. Kählert, C. Arran, and M. Bonitz, Plasma Phys Controlled Fusion 57, 025004 (2015).

${ }^{23}$ W. J. Miloch, M. Kroll, and D. Block, Phys. Plasmas 17, 103703 (2010).

${ }^{24}$ W. Miloch, D. Darian, and M. Mortensen, Phys. Scr. 92, 114006 (2017).

${ }^{25}$ W. J. Miloch, H. Jung, D. Darian, F. Greiner, M. Mortensen, and A. Piel, New J. Phys. 20, 073027 (2018).

${ }^{26}$ J. Carstensen, F. Greiner, and A. Piel, Phys. Rev. Lett. 109, 135001 (2012).

${ }^{27}$ E. Thomas, U. Konopka, R. L. Merlino, and M. Rosenberg, Phys. Plasmas 23, 055701 (2016).

${ }^{28}$ H. Jung, F. Greiner, O. H. Asnaz, and A. Piel, Phys. Plasmas 22, 053702 (2015).

${ }^{29}$ H. Jung, F. Greiner, A. Piel, and W. J. Miloch, Phys. Plasmas 25, 073703 (2018).

${ }^{30}$ A. Piel, H. Jung, and F. Greiner, Phys. Plasmas 25, 083703 (2018) 
${ }^{31}$ T. Nitter, Plasma Sources Sci. Technol. 5, 93 (1996).

${ }^{32}$ J. E. Allen, Phys. Scr. 45, 497 (1992).

${ }^{33}$ M. S. Barnes, J. H. Keller, J. C. Forster, J. A. O'Neill, and D. K. Coultas, Phys. Rev. Lett. 68, 313 (1992).

${ }^{34}$ C. K. Birdsall and A. B. Langdon, Plasma Physics Via Computer Simulation (Adam Hilger, Bristol, 1991).
${ }^{35}$ L. Nyland, M. Harris, and J. Prins, in GPU Gems 3, edited by H. Nguyen (Pearson Education, Inc., Upper Saddle River, NJ, 2008), Chap. 31.

${ }^{36}$ A. Piel and J. Goree, Phys. Rev. E 88, 063103 (2013).

${ }^{37}$ I. H. Hutchinson, Plasma Phys. Controlled Fusion 44, 1953 (2002).

${ }^{38}$ L. Patacchini, I. H. Hutchinson, and G. Lapenta, Phys. Plasmas 14, 062111 (2007). 